\title{
Next-to-Next-to-Leading Order Mixed QCD-Electroweak Corrections to On-Shell $Z$ Production
}

\author{
Roberto Bonciani, ${ }^{1,2, *}$ Federico Buccioni $\oplus^{3, \dagger}$ Narayan Rana ${ }^{4,+}{ }^{4,+}$ and Alessandro Vicini $\oplus^{5,4, \S}$ \\ ${ }^{1}$ Università di Roma "La Sapienza," Piazzale Aldo Moro 5, 00185 Roma, Italy \\ ${ }^{2}$ INFN Sezione di Roma, Piazzale Aldo Moro 5, 00185 Roma, Italy \\ ${ }^{3}$ Rudolf Peierls Centre for Theoretical Physics, Clarendon Laboratory, Parks Road, Oxford OX1 3PU, United Kingdom \\ ${ }^{4}$ INFN Sezione di Milano, Via Celoria 16, 20133 Milano, Italy \\ ${ }^{5}$ Dipartimento di Fisica "Aldo Pontremoli," University of Milano, Via Celoria 16, 20133 Milano, Italy
}

(Received 18 July 2020; revised 12 October 2020; accepted 29 October 2020; published 2 December 2020)

\begin{abstract}
We present the first analytical results for the $\mathcal{O}\left(\alpha \alpha_{s}\right)$ corrections to the total cross section for the inclusive production of an on-shell $Z$ boson at hadron colliders. We include the complete set of contributions, with photonic and massive weak gauge boson effects, which have been computed in analytical form and expressed in terms of polylogarithmic and elliptic integrals. We present numerical results, relevant for the precision studies at the LHC. These corrections increase the accuracy of the predictions and contribute to the reduction of the QCD component of the theoretical uncertainty.
\end{abstract}

DOI: 10.1103/PhysRevLett.125.232004

The production in hadronic collisions of a pair of leptons, each with large transverse momentum, is known as the Drell-Yan (DY) process and it plays a fundamental role for our understanding of quantum chromodynamics (QCD) as the theory of the strong interactions. The lepton pair acts as a probe of the initial-state proton structure: it allows for the measurement of the proton collinear parton density functions (PDFs) and for the study of the QCD dynamics from the analysis of the lepton-pair transverse momentum distribution. The kinematical distributions of the final-state leptons allow for precision tests of the electroweak (EW) standard model (SM), with the determination of the weak mixing angle and of the masses $m_{W, Z}$ and decay widths $\Gamma_{W, Z}$ of the $W$ and $Z$ bosons.

The production of an on-shell $Z$ boson represents a special kinematical configuration of the full neutral-current DY process. This process is described in lowest order by quark-antiquark annihilation into a $Z$ boson via $\mathrm{EW}$ interaction. The evaluation of the next-to-leading order (NLO) [1], next-to-next-to-leading order (NNLO) [2,3], and next-to-next-to-next-to-leading order $\left(\mathrm{N}^{3} \mathrm{LO}\right)$ [4,5] QCD corrections to the production of an on-shell gauge boson, supplemented by the resummation of the logarithmically enhanced terms due to soft gluon emission [6-12], allows for the accurate estimate of the total cross section, the reduction of the impact of QCD uncertainty, and the

Published by the American Physical Society under the terms of the Creative Commons Attribution 4.0 International license. Further distribution of this work must maintain attribution to the author(s) and the published article's title, journal citation, and DOI. Funded by SCOAP. precise assessment of its actual size. The best available result for the inclusive production of a virtual photon includes up to $\mathrm{N}^{3} \mathrm{LO}$ QCD corrections [13]. It shows a dependence on the QCD renormalization $\left(\mu_{R}\right)$ and factorization $\left(\mu_{F}\right)$ scale choices at the subpercent level for virtualities $Q>70 \mathrm{GeV}$ and at the percent level for smaller $Q$ values, with a stronger sensitivity to the choice of the factorization scale.

In this high-precision QCD framework, the inclusion of EW effects becomes mandatory. The NLO-EW corrections to the DY process have been computed in Refs. [14-18] and are comparable in size to the NNLO-QCD effects. The theoretical uncertainty associated with missing higherorder EW corrections is formally at the NNLO-EW level and it is significantly reduced compared to the leading order (LO) case. Using different input parameters as a mean to estimate the size of missing higher-order EW effects, one finds that the $\mathrm{LO}$ variation is at the $\mathcal{O}(3.5 \%)$ level, whereas the NLO-EW one is reduced down to the $\mathcal{O}(0.5 \%)$ level. The higher-order QCD predictions are only LO accurate from the point of view of the EW interaction, thus they suffer from the uncertainty associated with different choices of input parameters. If we consider the NNLOQCD prediction, supplemented with the NLO-EW one, we find a scheme uncertainty at the $\mathcal{O}(0.88 \%)$ level. This value is significant for any precision test and comparable to the residual QCD uncertainty. On the other hand, a specular discussion applies to the NLO-EW corrections, which are only LO from the point of view of the strong interaction. They suffer from large uncertainties under variations of the factorization scale. The canonical $\mu_{F}$ variation by a factor 2 about its central value yields a change of the LO cross section by $\pm 18 \%$ and, in turn, a change of the NLO-EW 
correction at the $\mathcal{O}(0.5 \%)$ level. In order to increase the control on the theoretical error, it is therefore mandatory to include in the analysis the mixed QCD-EW corrections, since they stabilize both the dependence on the QCD scales of the higher-order EW corrections and the dependence on the EW input parameters of the higher-order QCD corrections.

The mixed corrections to the DY process in the resonance region have been studied in the so-called pole approximation in Refs. [19,20], where QCD and EW effects are factorized between production and decay of the vector boson. As far as the production of an on-shell $Z$ boson is concerned, the exact QCD-QED corrections have been considered in Refs. [21-23], while in Ref. [24] we have computed the EW effects in the $q \bar{q}$ initiated channels. The complete set of QCD $\times \mathrm{EW}$ effects have been presented in Ref. [25] at the fully differential level, using a combination of analytical and numerical techniques. As for the off-shell neutral-current DY process, the QCD $\times$ QED corrections to the production of a pair of neutrinos have been discussed in Ref. [26].

In this Letter we present the totally inclusive cross section for a single on-shell $Z$ boson production in hadron-hadron collisions, including the $\mathcal{O}\left(\alpha \alpha_{s}\right)$ mixed QCD-EW corrections stemming from all the relevant partonic channels. The inclusion of the $\mathcal{O}\left(\alpha \alpha_{s}\right)$ corrections increases the accuracy of the prediction and it reduces the impact of the residual theoretical uncertainties. The results have been fully computed in analytical form and expressed in terms of polylogarithms and elliptic integrals, requiring the evaluation of new two-loop master integrals (MIs) that were not available in the literature.

Theoretical framework.-At hadron colliders, the inclusive production cross section $\sigma_{\text {tot }}$ of an on-shell $Z$ boson $(p p \rightarrow Z+X)$ is written, based on the factorization theorem, as

$$
\sigma_{\mathrm{tot}}(\tau)=\sum_{i, j \in q, \bar{q}, g, \gamma} \int d x_{1} d x_{2} f_{i}\left(x_{1}\right) f_{j}\left(x_{2}\right) \sigma_{i j}(z),
$$

where the sum runs over all possible initial state partons, namely quarks, gluons, and photons. The ratios $\tau=m_{Z}^{2} / S$ and $z=m_{Z}^{2} / \hat{s}$ compare the $Z$ boson mass, $m_{Z}$, with $S$ and $\hat{s}$, the hadronic and partonic center of mass energy squared, respectively. The variables $S$ and $\hat{s}$ are related by $\hat{s}=x_{1} x_{2} S$ through the Bjorken momentum fractions $x_{1}, x_{2}$. The hadron-level result is obtained via the convolution of the cross section $\sigma_{i j}$ of the partonic process $i j \rightarrow Z+X$ with the physical parton densities $f_{i}(x)$. The cross sections are inclusive over the additional partons radiated present in the final state and collectively indicated as $X$. However, we do not include the processes with the emission of one extra massive on-shell gauge boson, as their measurement depends on the details of the experimental event selection. The process that contributes at the lowest perturbative order
(Born approximation) is quark-antiquark annihilation, while additional channels open in higher perturbative orders. We include all the corrections of $\mathcal{O}\left(\alpha \alpha_{s}\right)$ relative to the Born process. Each partonic cross section admits a double expansion in the electromagnetic and strong coupling constants, $\alpha$ and $\alpha_{s}$, respectively. At NNLO-QCD $\times$ EW we need to consider two-loop virtual corrections to the $q \bar{q} \rightarrow Z$ process (dubbed double virtual), single virtual corrections to the processes with one additional parton in the final state (dubbed real virtual), and the $\mathcal{O}\left(\alpha \alpha_{s}\right)$ part [27] of the tree-level cross section of the processes with two additional real partons in the final state (dubbed double real). The charged-current weak interaction couples up- and down-type fermions, so that it is natural to observe a change of flavor in the internal lines when a $W$ boson is exchanged, but also down-type quarks in the final state of a process initiated by up-type quarks (and vice versa). Their impact eventually depends on the values of the $Z$ coupling to the different flavors, in such a way that their complete evaluation requires the inclusion of one complete quark doublet in the hadron-level cross section. In the present study we consider a diagonal Cabibbo-KobayashiMaskawa (CKM) matrix and our predictions are given in the four-flavor scheme, with the first two fermionic families.

The prediction of the hadron-level cross section requires us to express the bare couplings and masses in terms of physical parameters via renormalization. The choice of the background field gauge (BFG) [28] allows us to restore the validity of $U(1)_{\mathrm{em}}$-like Ward identities between the vertex corrections and the external quark wave function corrections in the full EW model. We explicitly verify these Ward identities at $\mathcal{O}\left(\alpha \alpha_{s}\right)$. If we choose to express $\left(g, g^{\prime}, v\right)$, the $S U(2)_{L}$ and $U(1)_{Y}$ couplings, and the Higgs field vacuum expectation value, in terms of $\left(G_{\mu}, m_{W}, m_{Z}\right)$ (dubbed $G_{\mu}$ scheme), where $G_{\mu}$ is the Fermi constant, then the weak charge renormalization is achieved by the replacement

$$
\frac{g_{0}}{c_{0}} Z_{Z Z}^{1 / 2} \rightarrow \sqrt{4 \sqrt{2} G_{\mu} m_{Z^{2}}}\left(1-\frac{1}{2} \Delta r+\frac{1}{2} \delta g_{Z}\right) .
$$

We denote with a 0 subscript all the bare quantities. We abbreviate with $c=m_{W} / m_{Z}$ the cosinus of the weak mixing angle $\left(s^{2}=1-c^{2}\right)$, and we define $\delta g_{Z} \equiv \delta Z_{Z Z}+$ $\delta e^{2} / e^{2}+\left(s^{2}-c^{2}\right) /\left(c^{2}\right)\left(\delta s^{2} / s^{2}\right)$ where $Z_{Z Z}=1+\delta Z_{Z Z}$ is the $Z Z$ wave function renormalization constant, $\Delta r$ is a finite correction [29] expressing the relation between the Fermi constant and the muon decay amplitude, $\delta s^{2}=c^{2}\left(\delta m_{Z}{ }^{2} / m_{Z}{ }^{2}-\delta m_{W}{ }^{2} / m_{W}{ }^{2}\right), \quad$ and $\delta e=e_{0}-e$, $\delta m_{W, Z}^{2}=m_{W, Z 0}^{2}-m_{W, Z}^{2}$ are the electric charge and gauge boson mass counterterms. The $\delta g_{Z}$ factor is, in the BFG, an UV finite correction. The $\Delta r$ parameter and the counterterms can be evaluated in perturbation theory and we keep terms of $\mathcal{O}(\alpha)$ and $\mathcal{O}\left(\alpha \alpha_{s}\right)$ [30,31]. For consistency, they have to be expressed in terms of $\left(G_{\mu}, m_{W}, m_{Z}\right)$. In addition 
to the redefinition of the overall weak coupling, a second renormalization correction modifies the vector coupling $v_{q}$ of the $Z$ boson to the quarks: $v_{q}=T_{3}^{(q)}-2 Q_{q} s^{2} \rightarrow T_{3}^{(q)}-$ $2 Q_{q}\left(s^{2}+\delta s^{2}+(c s / 2) \delta Z_{A Z}\right)$, with $\delta Z_{A Z}$ the renormalization constant of the $\gamma-Z$ mixing. In the BFG also this shift of $v_{q}$ is UV finite. If we instead choose to relate $\left(g, g^{\prime}, v\right)$ to the $\left(\alpha, m_{W}, m_{Z}\right)$ set of inputs [dubbed $\alpha(0)$ scheme], the replacement of the overall coupling is $g_{0} / c_{0} Z_{Z Z}^{1 / 2} \rightarrow$ $\sqrt{4 \pi \alpha} /(s c)\left(1+\frac{1}{2} \delta g_{Z}\right)$, while the redefinition of the vector coupling remains the same as in the other scheme. The $\alpha(0)$-scheme choice is historically [29,32] one of the simplest EW renormalization input schemes [33], but the low scale at which the fine structure constant is measured yields in turn the appearance of large logarithmic corrections in the perturbative expansion. The results depend on the value of the light-quark masses or, alternatively, on an experimental input $\Delta \alpha_{\text {had }}\left(m_{Z}\right)$ [37-40] needed to evaluate the hadronic contribution to the running of the electromagnetic coupling at low scales. The $G_{\mu}$ scheme is the most commonly adopted at hadron colliders, because it reabsorbs in its definition large logarithmic corrections and does not depend on the value of the light quark masses. We take both as two extreme input possibilities and we use the difference between the corresponding predictions as a conservative estimate of the size of the missing EW higher order effects. We remark that the size of the NLO-EW correction in the $G_{\mu}$ scheme is smaller than in the $\alpha(0)$ case because of an accidental partial cancellation between $\delta g_{Z}$ and $\Delta r$. This fact is welcome in view of the phenomenological studies, but it should be taken with care when estimating the size of the residual theoretical uncertainties.

The hadron-level cross section requires us to reabsorb the initial state singularities in the definition of the physical proton PDFs. Starting from NLO-EW, we need to subtract also the QED initial state collinear singularities, to reabsorb them in the proton PDFs, and to evolve the latter with Dokshitzer-Gribov-Lipatov-Altarelli-Parisi (DGLAP) equations that include a QED kernel. The presence of a photon density in the proton yields new partonic scattering processes. The hadron-level cross section is thus given by an extended set of processes, with respect to those appearing in the QCD higher-order corrections. The subtraction kernels at $\mathcal{O}\left(\alpha \alpha_{s}\right)$ are based on the splitting functions computed in Ref. [41], and allow us to cancel from the partonic cross sections all the initial-state collinear singularities. Two recent sets of proton PDFs accounting for NLO-EW corrections in the analysis and evolved with a DGLAP QED kernel have been discussed in Refs. [42,43]. These parameterizations have been obtained, starting from the same dataset, with two distinct modeling hypotheses, QCD only or full QCD-QED interaction, and allow a meaningful comparison of the corresponding results. We remark in fact that we have only two consistent options to compute the best prediction for the total cross section: (i) including only QCD radiative corrections and using
PDFs evolved with only-QCD DGLAP kernels; (ii) including QCD and EW corrections convoluted with QCD-QED PDFs. Given the impact of the EW corrections, we consider the second option the only alternative to compute the best prediction for this cross section.

Computational details. - In this Letter, we extend the studies presented in Refs. [24,44], completing the analytical evaluation of all the $\mathcal{O}\left(\alpha \alpha_{s}\right)$ contributions to the inclusive cross section for on-shell $Z$ boson production. These entail two-loop integrals in the double-virtual corrections, one-loop integrals integrated over a two-body phase-space in the realvirtual case, and three-body phase-space integrals for the double-real one. In the last two cases we use the reverse unitarity method $[45,46]$ in order to treat phase-space integrals on the same footing as loop integrals. This makes it possible to exploit the techniques developed for the computation of multiloop integrals, namely integration-byparts identities [47-49] for integral reduction, through public computer programs [50-54], and the method of differential equations [55-61] to compute the MIs.

The massless MIs are the same as presented in Ref. [45]. The relevant two-loop MIs with one external $Z$ boson and internal massive lines were studied in Refs. [62-69]. We performed an independent calculation of these contributions, finding complete agreement with the literature. The corresponding results for the real-virtual and the doublereal cases were not available in the literature. From a technical stand point these constitute the most involved part of the calculation. In particular, the analytic computation of the integrals in special kinematic limits which serve as boundary conditions to the system of differential equations.

The two-loop contributions are proportional to $\delta(1-z)$, and the relevant constants have been computed from the onshell limit of the MIs. The real-virtual and double-real contributions are expressed in terms of $\delta(1-z)$, plus distributions and generalized polylogarithms (GPLs) [70-72] or cyclotomic harmonic polylogarithms [73]. This allows the use of publicly available computer programs [74-79] for the manipulation and efficient numerical evaluation of our final results. However, we point out that a set of three MIs arises in the double-real corrections, the solutions of which are given in terms of elliptic functions. We solved the corresponding differential equations as a series expansions around $z=0, z=1$ and intermediate values allowing for a reliable numerical evaluation [80].

The calculation of the MIs that depend on two different masses $\left(m_{Z}\right.$ and $\left.m_{W}\right)$ is done performing an expansion of the integrand in powers of the ratio $\delta_{m^{2}}=\left(m_{Z}^{2}-m_{W}^{2}\right) / m_{Z}^{2}$. The coefficients of such expansion can be expressed, therefore, as a combination of the equal-mass MIs. While for the double-virtual corrections the expansion in $\delta_{m^{2}}$ is not strictly necessary, since the knowledge of the MIs for an off-shell $Z$ boson allows for an exact calculation with $m_{W} \neq m_{Z}$, the reduction of one mass scale in the computation of the real emission processes reduces effectively the 
complication of the calculation. The results, presented in this Letter, are obtained using the expansion up to second order in $\delta_{m^{2}}$, wherever needed. We stress that the couplings of the $Z$ boson to fermions are expressed in terms of the physical value of the weak mixing angle $\sin ^{2} \theta_{W}=1-m_{W}^{2} / m_{Z}^{2}$.

Results.-In this section we present the numerical results for the inclusive total cross section for the production of an on-shell $Z$ boson in proton-proton collisions at the LHC. They are computed using the following values of the input parameters: $\sqrt{S}=13 \mathrm{TeV}, m_{H}=125.0 \mathrm{GeV}$, $m_{W}=80.358 \mathrm{GeV}, m_{Z}=91.153 \mathrm{GeV}, m_{t}=173.2 \mathrm{GeV}$, $\alpha^{-1}=137.035999074, G_{\mu}=1.166378110^{-5} \mathrm{GeV}^{-2}$ and $\Delta \alpha_{\text {had }}\left(m_{Z}\right)=0.027572$ as determined in Ref. [37], where $m_{t}$ and $m_{H}$ are the top quark and Higgs boson masses. To present the results, we arrange $\sigma_{\text {tot }}$ as follows:

$$
\sigma_{\mathrm{tot}}=\sigma_{\mathrm{LO}}+\sigma_{10}+\sigma_{01}+\sigma_{11}+\sigma_{20}
$$

where $\sigma_{i j}$ indicates the sole contribution from the relative perturbative order $\mathcal{O}\left(\alpha_{s}^{i} \alpha^{j}\right)$ with respect to the Born. We define the combinations

$$
\begin{aligned}
& B_{1}=\sigma_{\mathrm{LO}}+\sigma_{10}+\sigma_{20}, \\
& B_{2}=\sigma_{\mathrm{LO}}+\sigma_{10}+\sigma_{01}+\sigma_{20}, \\
& B_{3}=\sigma_{\mathrm{LO}}+\sigma_{10}+\sigma_{01}+\sigma_{11}+\sigma_{20}, \\
& B_{3}^{\gamma}=\sigma_{\mathrm{LO}}+\sigma_{10}+\sigma_{01}+\sigma_{11}^{\gamma}+\sigma_{20},
\end{aligned}
$$

to study the effect of individual contributions. We note that $\sigma_{11}^{\gamma}$ denotes the mixed NNLO-QCD $\times$ QED corrections whereas $\sigma_{11}$ denotes the full QCD-EW set. All the $B_{i}$ 's are computed with the NNPDF31_nnlo_as_0118 luxqed nf 4 [42] proton PDF set, which is evolved with both $\mathrm{QCD}$ and QED kernels and has a photon density derived according to the LUX-QED model $[87,88]$. We also define $A_{1}$ as the quantity corresponding to $B_{1}$ but evaluated with the NNPDF31_nnlo_as_0118_nf_4 PDF set, the version with only-QCD DGLAP evolution. We use both the LHAPDF-6 [89] and HOPPET [90] to interface the PDFs. The relevant value of the strong coupling constant is $\alpha_{s}\left(m_{Z}\right)=0.1127$.

In Table I, we show the results at different perturbative orders for the cross section, in the $G_{\mu}$ and $\alpha(0)$ schemes. $A_{1}$ is only LO from the EW point of view and, as a consequence, the two predictions differ by $3.53 \%$. The inclusion in $B_{2}$ of the NLO-EW corrections reduces the spread to $0.88 \%$. The complete NNLO-QCD $\times$ EW corrections in $B_{3}$ further reduce this uncertainty down to the $0.23 \%$ level. The comparison in the $G_{\mu}$ scheme between the QCD-only prediction $A_{1}$ and the best QCD-EW predictions $B_{3}\left(B_{3}^{\gamma}\right)$ shows that the latter reduce the value of the cross section by $-0.57 \%(-0.49 \%)$. For the sake of a technical
TABLE I. Comparison of the cross sections (expressed in $\mathrm{pb}$ ) computed at different orders, in the $G_{\mu}$ and $\alpha(0)$ input schemes setting the factorization and renormalization scales as $\mu_{R}=\mu_{F}=m_{Z}$.

\begin{tabular}{lccc}
\hline \hline Order & $G_{\mu}$ & $\alpha(0)$ & $\delta_{G_{\mu}-\alpha(0)}(\%)$ \\
\hline$A_{1}$ & 55787 & 53884 & 3.53 \\
$B_{1}$ & 55651 & 53753 & 3.53 \\
$B_{2}$ & 55501 & 55015 & 0.88 \\
$B_{3}^{\gamma}$ & 55516 & 55029 & 0.88 \\
$B_{3}$ & 55469 & 55340 & 0.23 \\
\hline \hline
\end{tabular}

comment, the comparison between $A_{1}$ and $B_{1}$ gives an estimate of the impact of DGLAP joint QCD and QED evolution on the quark and gluon densities, yielding a reduction of the cross section by $-0.24 \%$. We stress that $B_{1}$ cannot be considered a physical prediction, because it lacks the EW corrections and the photon-induced partonic processes. The size of $\sigma_{11}^{\gamma}$ is $0.03 \%$ of the Born, while $\sigma_{11}$ is negative and larger than $\sigma_{11}^{\gamma}$ by almost a factor of 3 , as also observed in Ref. [25].

In Table II we show the results obtained under variation of the factorization scale $\mu_{F}=\xi_{F} m_{Z}$ in the range given by $\xi_{F}=\left(\frac{1}{2}, 1,2\right)$, keeping $\mu_{R}=m_{Z}$. Since the NLO-EW corrections present in $B_{2}$ are only LO from the QCD point of view, they vary accordingly with $\mu_{F}$, while the inclusion of the NNLO-QCD $\times \mathrm{EW}$ terms in $B_{3}$ stabilizes the results. The improvement from $B_{2}$ to $B_{3}$ is more evident in the $\alpha(0)$ scheme, where the size of the NLO-EW corrections is larger than in the $G_{\mu}$ scheme. The $A_{1}$ prediction has a variation by $+0.26 \%,-0.99 \%$, which increases in the $B_{2}$ case to $+0.52 \%,-1.26 \%$. The improvement induced by the mixed QCD $\times \mathrm{EW}$ corrections in $B_{3}$ brings the uncertainty down to $+0.37 \%,-1.13 \%$.

In Table III we show the results obtained under variation of the renormalization scale $\mu_{R}=\xi_{R} m_{Z}$ in the range given by $\xi_{R}=\left(\frac{1}{2}, 1,2\right)$, keeping $\mu_{F}=m_{Z}$. In both cases, $\xi_{R}=2$ and $\xi_{R}=1 / 2$, there is a mild reduction of the cross section with respect to the central $\xi_{R}=1$ value. This decrease is of almost $-0.14 \%$ in both the $G_{\mu}$ scheme and $\alpha(0)$ scheme.

Evaluating the $\mu_{F}$ and $\mu_{R}$ dependence through the 7point scale variation, we obtain our best prediction for $B_{3}$, which reads $55469_{-1.01 \%}^{+0.65 \%} \mathrm{pb}$ and $55340_{-1.13 \%}^{+0.68 \%} \mathrm{pb}$, in the $G_{\mu}$ and $\alpha(0)$ schemes, respectively.

TABLE II. Dependence of the cross sections, expressed in pb and computed at different perturbative orders, under variation of the factorization scale $\mu_{F}$, keeping $\mu_{R}=m_{Z}$.

\begin{tabular}{lcc}
\hline \hline & $G_{\mu}$ scheme & $\alpha(0)$ scheme \\
\hline$A_{1}$ & $55787_{-0.99 \%}^{+0.26 \%}$ & $53884_{-0.99 \%}^{+0.26 \%}$ \\
$B_{2}$ & $55501_{-0.99 \%}^{+0.26 \%}$ & $55015_{-1.26 \%}^{+0.52 \%}$ \\
$B_{3}$ & $55469_{-1.01 \%}^{+0.28 \%}$ & $55340_{-1.13 \%}^{+0.37 \%}$ \\
\hline \hline
\end{tabular}


TABLE III. Dependence of the cross sections, expressed in $\mathrm{pb}$ and computed at different perturbative orders, under variation of the renormalization scale $\mu_{R}$, keeping $\mu_{F}=m_{Z}$.

\begin{tabular}{lcc}
\hline \hline & $G_{\mu}$ scheme & $\alpha(0)$ scheme \\
\hline$A_{1}$ & $55787_{-0.13 \%}^{-0.15 \%}$ & $53884_{-0.13 \%}^{-0.15 \%}$ \\
$B_{2}$ & $55501_{-0.12 \%}^{-0.15 \%}$ & $55015_{-0.12 \%}^{-0.15 \%}$ \\
$B_{3}$ & $55469_{-0.13 \%}^{-0.15 \%}$ & $55340_{-0.05 \%}^{-0.21 \%}$ \\
\hline \hline
\end{tabular}

We have checked analytically and numerically the $\mathrm{QCD} \times \mathrm{QED}$ part of our result against the one in Ref. [21], finding complete agreement. We have checked numerically our inclusive cross section against the results presented in Ref. [25], finding agreement within the expected numerical accuracy.

In conclusion, we have presented the prediction of the on-shell $Z$ inclusive total production cross section, including the full set of $\mathcal{O}\left(\alpha \alpha_{s}\right)$ corrections. The evaluation of the cross section requires the consistent usage of proton PDFs which are extracted and evolved including QED effects. The comparison with the NNLO-QCD predictions, based on only-QCD proton PDFs, shows a change of the central value by $-0.57 \%$. The NNLO-QCD $\times \mathrm{EW}$ corrections reduce the impact of two sources of theoretical uncertainty, which were not previously under control: the input parameters and the factorization scale variation uncertainties. The increased precision of the prediction of the $Z$-production cross section may have an impact on the determination of the proton PDFs and on the hadron collider luminosity studies.

We would like to thank F. Caola, M. Delto, P. K. Dhani, M. Jaquier, J.-N. Lang, K. Melnikov, V. Ravindran, and R. Röntsch for useful discussions. A. V. is supported by the Italian Ministero della Università e della Ricerca (Grant No. PRIN2017) and by the European Research Council under the European Unions Horizon 2020 research and innovation Programme (Grant Agreement No. 740006). R. B. is partly supported by the Italian Ministero della Università e della Ricerca (MIUR) under Grant No. PRIN 20172LNEEZ. The research of F. B. was partially supported by the ERC Starting Grant No. 804394 HIPQCD. R. B. and N. R. acknowledge the COST (European Cooperation in Science and Technology) Action CA16201 PARTICLEFACE for partial support.

*roberto.bonciani@ roma1.infn.it

†federico.buccioni@physics.ox.ac.uk

\#narayan.rana@mi.infn.it

\$alessandro.vicini@mi.infn.it

[1] G. Altarelli, R. K. Ellis, and G. Martinelli, Nucl. Phys. B157, 461 (1979).

[2] R. Hamberg, W. van Neerven, and T. Matsuura, Nucl. Phys. B359, 343 (1991).
[3] R. V. Harlander and W. B. Kilgore, Phys. Rev. Lett. 88, 201801 (2002).

[4] T. Ahmed, M. Mahakhud, N. Rana, and V. Ravindran, Phys. Rev. Lett. 113, 112002 (2014).

[5] Y. Li, A. von Manteuffel, R. M. Schabinger, and H. X. Zhu, Phys. Rev. D 90, 053006 (2014).

[6] G. F. Sterman, Nucl. Phys. B281, 310 (1987).

[7] S. Catani and L. Trentadue, Nucl. Phys. B327, 323 (1989).

[8] S. Catani and L. Trentadue, Nucl. Phys. B353, 183 (1991).

[9] S. Moch and A. Vogt, Phys. Lett. B 631, 48 (2005).

[10] V. Ravindran, Nucl. Phys. B752, 173 (2006).

[11] S. Catani, L. Cieri, D. de Florian, G. Ferrera, and M. Grazzini, Nucl. Phys. B888, 75 (2014).

[12] A. H. Ajjath, G. Das, M. C. Kumar, P. Mukherjee, V. Ravindran, and K. Samanta, J. High Energy Phys. 10 (2020) 153.

[13] C. Duhr, F. Dulat, and B. Mistlberger, Phys. Rev. Lett. 125, 172001, (2020).

[14] U. Baur, O. Brein, W. Hollik, C. Schappacher, and D. Wackeroth, Phys. Rev. D 65, 033007 (2002).

[15] C. Carloni Calame, G. Montagna, O. Nicrosini, and A. Vicini, J. High Energy Phys. 10 (2007) 109.

[16] A. Arbuzov, D. Bardin, S. Bondarenko, P. Christova, L. Kalinovskaya, G. Nanava, and R. Sadykov, Eur. Phys. J. C 54, 451 (2008).

[17] S. Dittmaier and M. Huber, J. High Energy Phys. 01 (2010) 060.

[18] L. Buonocore, M. Grazzini, and F. Tramontano, Eur. Phys. J. C 80, 254 (2020).

[19] S. Dittmaier, A. Huss, and C. Schwinn, Nucl. Phys. B885, 318 (2014).

[20] S. Dittmaier, A. Huss, and C. Schwinn, Nucl. Phys. B904, 216 (2016).

[21] D. de Florian, M. Der, and I. Fabre, Phys. Rev. D 98, 094008 (2018).

[22] L. Cieri, G. Ferrera, and G. F. R. Sborlini, J. High Energy Phys. 08 (2018) 165.

[23] M. Delto, M. Jaquier, K. Melnikov, and R. Röntsch, J. High Energy Phys. 01 (2020) 043.

[24] R. Bonciani, F. Buccioni, N. Rana, I. Triscari, and A. Vicini, Phys. Rev. D 101, 031301 (2020).

[25] F. Buccioni, F. Caola, M. Delto, M. Jaquier, K. Melnikov, and R. Röntsch, arXiv:2005.10221.

[26] L. Cieri, D. de Florian, M. Der, and J. Mazzitelli, J. High Energy Phys. 09 (2020) 155..

[27] The tree-level amplitude of some partonic processes is mediated by the exchange of both QCD and EW bosons, so that it simultaneously contributes to different perturbative orders. We isolate the $\mathcal{O}\left(\alpha \alpha_{s}\right)$ terms.

[28] A. Denner, G. Weiglein, and S. Dittmaier, Nucl. Phys. B440, 95 (1995).

[29] A. Sirlin, Phys. Rev. D 22, 971 (1980).

[30] B. A. Kniehl, Nucl. Phys. B347, 86 (1990).

[31] G. Degrassi and A. Vicini, Phys. Rev. D 69, 073007 (2004).

[32] A. Denner, Fortschr. Phys. 41, 307 (1993).

[33] The $\left(\alpha(0), G_{\mu}, m_{Z}\right)$ input scheme has been used at LEP, and recently the complete NNLO-EW corrections to the full set of EW observables at the $Z$ resonance have been presented in Refs. [34,35]. However, at hadron colliders, input 
schemes including $m_{W}$ are preferable in view of its precision determination (cf. also Ref. [36]).

[34] I. Dubovyk, A. Freitas, J. Gluza, T. Riemann, and J. Usovitsch, Phys. Lett. B 783, 86 (2018).

[35] I. Dubovyk, A. Freitas, J. Gluza, T. Riemann, and J. Usovitsch, J. High Energy Phys. 08 (2019) 113.

[36] A. Denner and S. Dittmaier, Phys. Rep. 864, 1 (2020).

[37] F. Jegerlehner, J. Phys. G 29, 101 (2003).

[38] F. Jegerlehner, Acta Phys. Pol. B 49, 1157 (2018).

[39] A. Keshavarzi, D. Nomura, and T. Teubner, Phys. Rev. D 97, 114025 (2018).

[40] M. Davier, A. Hoecker, B. Malaescu, and Z. Zhang, Eur. Phys. J. C 80, 241 (2020); 80, 410(E) (2020).

[41] D. de Florian, G. F. R. Sborlini, and G. Rodrigo, Eur. Phys. J. C 76, 282 (2016).

[42] R. D. Ball et al. (NNPDF Collaboration), Eur. Phys. J. C 77, 663 (2017).

[43] L. Harland-Lang, A. Martin, R. Nathvani, and R. Thorne, Eur. Phys. J. C 79, 811 (2019).

[44] R. Bonciani, F. Buccioni, R. Mondini, and A. Vicini, Eur. Phys. J. C 77, 187 (2017).

[45] C. Anastasiou and K. Melnikov, Nucl. Phys. B646, 220 (2002).

[46] C. Anastasiou, S. Buehler, C. Duhr, and F. Herzog, J. High Energy Phys. 11 (2012) 062.

[47] F. Tkachov, Phys. Lett. 100B, 65 (1981).

[48] K. Chetyrkin and F. Tkachov, Nucl. Phys. B192, 159 (1981).

[49] S. Laporta, Int. J. Mod. Phys. A 15, 5087 (2000).

[50] P. Maierhöfer, J. Usovitsch, and P. Uwer, Comput. Phys. Commun. 230, 99 (2018).

[51] R. N. Lee, arXiv:1212.2685.

[52] R. N. Lee, J. Phys. Conf. Ser. 523, 012059 (2014).

[53] C. Studerus, Comput. Phys. Commun. 181, 1293 (2010).

[54] A. von Manteuffel and C. Studerus, arXiv:1201.4330.

[55] A. Kotikov, Phys. Lett. B 254, 158 (1991).

[56] E. Remiddi, Nuovo Cimento A 110, 1435 (1997).

[57] T. Gehrmann and E. Remiddi, Nucl. Phys. B580, 485 (2000).

[58] M. Argeri and P. Mastrolia, Int. J. Mod. Phys. A 22, 4375 (2007)

[59] J. M. Henn, J. Phys. A 48, 153001 (2015).

[60] J. Ablinger, A. Behring, J. Blümlein, A. De Freitas, A. von Manteuffel, and C. Schneider, Comput. Phys. Commun. 202, 33 (2016).

[61] J. Ablinger, J. Blümlein, P. Marquard, N. Rana, and C. Schneider, Nucl. Phys. B939, 253 (2019).

[62] J. Fleischer, A. Kotikov, and O. Veretin, Phys. Lett. B 417, 163 (1998).

[63] J. Fleischer, A. V. Kotikov, and O. L. Veretin, Nucl. Phys. B547, 343 (1999).
[64] U. Aglietti and R. Bonciani, Nucl. Phys. B668, 3 (2003).

[65] U. Aglietti and R. Bonciani, Nucl. Phys. B698, 277 (2004).

[66] U. Aglietti, R. Bonciani, G. Degrassi, and A. Vicini, Phys. Lett. B 600, 57 (2004).

[67] U. Aglietti, R. Bonciani, L. Grassi, and E. Remiddi, Nucl. Phys. B789, 45 (2008).

[68] R. Bonciani, G. Degrassi, and A. Vicini, Comput. Phys. Commun. 182, 1253 (2011).

[69] A. Kotikov, J. H. Kuhn, and O. Veretin, Nucl. Phys. B788, 47 (2008).

[70] A. Goncharov, Proc. Int. Cong. Math. 12, 374 (1995).

[71] A. B. Goncharov, arXiv:math/0103059.

[72] E. Remiddi and J. Vermaseren, Int. J. Mod. Phys. A 15, 725 (2000).

[73] J. Ablinger, J. Blümlein, and C. Schneider, J. Math. Phys. (N.Y.) 52, 102301 (2011).

[74] C. W. Bauer, A. Frink, and R. Kreckel, J. Symb. Comput. 33, 1 (2002).

[75] J. Vollinga and S. Weinzierl, Comput. Phys. Commun. 167, 177 (2005).

[76] J. Ablinger, Ph.D. thesis, Linz University, 2009 [arXiv:1011.1176].

[77] J. Ablinger, J. Blümlein, and C. Schneider, J. Math. Phys. (N.Y.) 54, 082301 (2013).

[78] J. Ablinger, Proc. Sci. LL2014 (2014) 019.

[79] L. Naterop, A. Signer, and Y. Ulrich, Comput. Phys. Commun. 253, 107165 (2020).

[80] Equivalent approaches have already been presented in Refs. [67,81-86]

[81] S. Pozzorini and E. Remiddi, Comput. Phys. Commun. 175, 381 (2006).

[82] J. Blümlein and C. Schneider, Phys. Lett. B 771, 31 (2017).

[83] R. N. Lee, A. V. Smirnov, and V. A. Smirnov, J. High Energy Phys. 03 (2018) 008.

[84] R. N. Lee, A. V. Smirnov, and V. A. Smirnov, J. High Energy Phys. 07 (2018) 102.

[85] R. Bonciani, G. Degrassi, P. P. Giardino, and R. Groeber, Comput. Phys. Commun. 241, 122 (2019).

[86] J. Blümlein, P. Marquard, N. Rana, and C. Schneider, Nucl. Phys. B949, 114751 (2019).

[87] A. Manohar, P. Nason, G. P. Salam, and G. Zanderighi, Phys. Rev. Lett. 117, 242002 (2016).

[88] A. V. Manohar, P. Nason, G. P. Salam, and G. Zanderighi, J. High Energy Phys. 12 (2017) 046.

[89] A. Buckley, J. Ferrando, S. Lloyd, K. Nordström, B. Page, M. Rüfenacht, M. Schönherr, and G. Watt, Eur. Phys. J. C 75, 132 (2015).

[90] G. P. Salam and J. Rojo, Comput. Phys. Commun. 180, 120 (2009). 